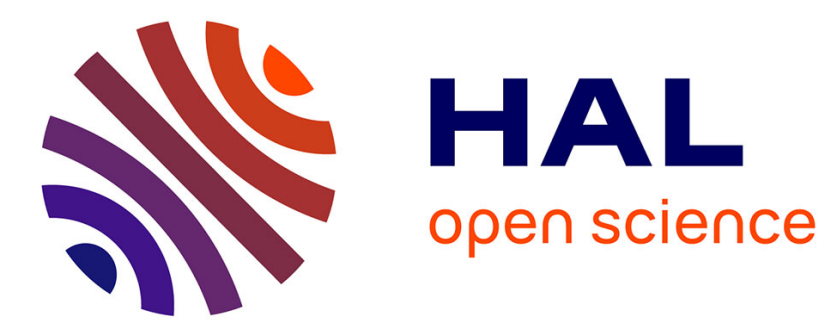

\title{
Le marché des égaux: un aspect socialiste de l'échange républicain
}

Vincent Bourdeau

\section{To cite this version:}

Vincent Bourdeau. Le marché des égaux: un aspect socialiste de l'échange républicain. Revue de Philosophie Economique / Review of Economic Philosophy, 2012, 13 (2), pp.3. 10.3917/rpec.132.0003 . hal-02302434

\section{HAL Id: hal-02302434 https://hal.science/hal-02302434}

Submitted on 1 Oct 2019

HAL is a multi-disciplinary open access archive for the deposit and dissemination of scientific research documents, whether they are published or not. The documents may come from teaching and research institutions in France or abroad, or from public or private research centers.
L'archive ouverte pluridisciplinaire HAL, est destinée au dépôt et à la diffusion de documents scientifiques de niveau recherche, publiés ou non, émanant des établissements d'enseignement et de recherche français ou étrangers, des laboratoires publics ou privés. 


\title{
LE MARCHÉ DES ÉGAUX : UN ASPECT SOCIALISTE DE L'ÉCHANGE RÉPUBLICAIN
}

\author{
Vincent Bourdeau
}

Vrin | «Revue de philosophie économique »

2012/2 Vol. 13 | pages 3 à 23

ISSN 1376-0971

ISBN 9782711652075

Article disponible en ligne à l'adresse :

https://www.cairn.inforevue-de-philosophie-economique-2012-2-page-3.htm

Distribution électronique Cairn.info pour Vrin.

(C) Vrin. Tous droits réservés pour tous pays.

La reproduction ou représentation de cet article, notamment par photocopie, n'est autorisée que dans les limites des conditions générales d'utilisation du site ou, le cas échéant, des conditions générales de la licence souscrite par votre établissement. Toute autre reproduction ou représentation, en tout ou partie, sous quelque forme et de quelque manière que ce soit, est interdite sauf accord préalable et écrit de l'éditeur, en dehors des cas prévus par la législation en vigueur en France. Il est précisé que son stockage dans une base de données est également interdit. 


\title{
Le marché des égaux : uN aspect sociaListe de L'échange RépubLicain
}

\author{
VINCENt BOURDEAU*
}

\begin{abstract}
Résumé
La défense du marché dans la tradition socialiste libérale est instrumentale: le marché est toléré lorsqu'il permet de produire des richesses en abondance que l'on peut redistribuer ex post. De son côté, la justification républicaine du marché, telle que présentée dans les travaux de Pettit, vise à réduire la domination dans l'échange. Cet article soutient qu'une telle justification nécessite d'être renforcée en l'adossant à un égalitarisme matériel, garant d'un échange entre égaux, établi ex ante.
\end{abstract}

Mots-Clefs : Pettit, Républicanisme, Marché, Egalitarisme matériel, Socialisme

\begin{abstract}
Summary
The defense of market economies in the liberal socialist tradition is largely instrumental: markets are tolerated insofar as they encourage enough circulation of wealth for some degree of ex post redistribution. On the other hand, the republican defense of market, as stated in Pettit's work, aims to reduce domination. This article argues that this defense could be strengthened by adding a material egalitarian condition as an ex ante guarantee for more equal forms of exchange.
\end{abstract}

Keywords: Pettit, Republicanism, Market, Material egalitarianism, Socialism

Classification JEL : B24, B51, D63, H82

* Université de Franche-Comté, 30 rue Mégevand, 25000 Besançon. E.A. 2274, Logiques de l'agir - contact : vincent.bourdeau@univ-fcomte.fr. Je tiens à remercier deux commentateurs anonymes de la revue pour les corrections et orientations suggérées en vue d'améliorer cet article. 
Deux courants en philosophie politique ont connu dans les années récentes un regain d'intérêt dans le public: le républicanisme, d'un côté, et le socialisme libéral, de l'autre ${ }^{1}$. L'un et l'autre mettent l'accent sur la promotion de l'individu en même temps qu'ils se présentent comme des alternatives à la critique marxienne du libéralisme. Ces deux courants ne sont pas pour autant superposables. Le républicanisme se distingue du socialisme libéral en ce qu'il insiste sur l'inscription de l'individu dans une communauté politique et sur le pouvoir que donne à l'individu cette inscription, tandis que le socialisme libéral défend la compatibilité de la liberté individuelle et de formes de redistribution de richesses que l'État doit prendre en charge pour améliorer le sort des plus désavantagés ${ }^{2}$. Le vieux dilemme entre "vertu civique » d'un côté et « justice sociale» de l'autre, mis en avant par les travaux de John Pocock (1997) à propos de l'époque moderne, se trouverait ainsi reconduit. D'un point de vue contemporain, ce dilemme renverrait à la capacité qu'aurait le socialisme libéral de s'attaquer aux impasses économiques de la modernité, tandis que le républicanisme de son côté n’offrirait pas véritablement de réponse à ces dernières ${ }^{3}$.

1. Pour un premier survol de la littérature sur le républicanisme aujourd'hui, voir: Pettit $(2007$; 2004), Spitz (2005), et sur le socialisme libéral : Canto-Sperber \& Urbinati (2003); Audier (2006). Les tentatives d'élaboration d'un «libéralisme égalitaire», depuis Rawls (1989) et sa Théorie de la justice, ont nettement dominé les débats en philosophie politique. Les développements post-rawlsiens pourraient être décrits dans les termes de l'alternative socialisme libéral / républicanisme que nous suivons ici. Deux compléments ont en effet été apportés à la théorie de Rawls : la mise en relation plus nette de l'égalitarisme et de la responsabilité individuelle d'un côté (Dworkin 2000) et la valorisation de l'autonomie politique et des « réalisations » humaines de l'autre (par exemple Fleurbaey 2006).

2. En ce sens, il s'agit bien d'un socialisme qui, si l'on en croit la définition classique donnée par E. Durkheim est moins un idéal civique ou politique qu'un idéal d'organisation économique de la société, de rationalisation de la décision économique - «On appelle socialiste toute doctrine qui réclame le rattachement de toutes les fonctions économiques, ou de certaines d'entre elles qui sont actuellement diffuses, aux centres directeurs et conscients de la société »- dont l'objectif est de « rendre moins mauvaise la condition du plus grand nombre» (Durkheim 1992, p. 49).

3. La relecture proposée par Audier du socialisme libéral semble plaider dans le sens de la clarification que je propose puisque très souvent, lorsqu'il s'agit de distinguer socialisme libéral historique et socialisme libéral actuel (dans sa version "troisième voie »), Audier a recours à l'inscription du premier dans un univers de valeurs républicain (Audier 2006, chap. 2). 
Dans cet article, je soutiendrai la thèse que le socialisme libéral n'est pas une doctrine unifiée et cohérente mais plutôt une étiquette qui permet de ranger sous un même nom un ensemble de mesures redistributives qui partagent une affinité d'esprit, à savoir produire une certaine protection sociale tout en respectant les libertés individuelles. Je défendrai l'idée que la théorie républicaine est en mesure d'offrir des justifications politiques plus solides à ces mesures, mais qu'elle ne peut le faire qu'en intégrant à ces justifications une dimension économique ${ }^{4}$, en particulier en dégageant une architecture spécifiquement républicaine du marché ${ }^{5}$. En rupture avec les thèses défendues par Pettit dans son article «Freedom in the Market » (Pettit 2006), je suggérerai que la poursuite de l'idéal républicain, c'est-à-dire la promotion de la liberté comme nondomination, nécessite d'intégrer aux conditions républicaines du marché une forme d'égalité matérielle assise sur une propriété publique des ressources naturelles se traduisant par un « revenu de citoyenneté ». Une telle architecture révèle une dimension socialiste de l'échange républicain ${ }^{6}$. L'égalité structurelle, égalité politique ou de statut, ne suffit pas à elle seule à éliminer les phénomènes de domination qui surviennent dans les échanges économiques, à moins qu'elle n'accueille en son sein une dimension d'égalité matérielle. En ce sens l'égalitarisme républicain doit être pluraliste, non seulement parce qu'il valorise d'autres valeurs que l'égalité mais

4. De ce point de vue, l'article de William H. Simon, «Social-Republican Property », est essentiel (1991). Dans cet article, je ne discuterai pas en détail l'ensemble de ce que cet auteur apporte au débat, en établissant une approche proprement républicaine des schèmes coopératifs en économie à partir d'une définition de la "propriété social-républicaine », je tenterai seulement de suggérer que la promotion d'une dimension publique de la propriété peut venir soutenir l'économie républicaine des coopératives qu'il promeut.

5. J'en reste, dans cet article, à un travail sur les justifications normatives d'une structure égalitariste matérielle du marché républicain, cherchant à mettre à jour celles qui sont les plus cohérentes eu égard à l'esprit même du néo-républicanisme pettitien. Je laisse de côté un travail qui consisterait à comprendre comment l'on passe de l'idéal du marché républicain ainsi défini à sa réalisation pratique.

6. L'idée de revenu d'existence ou allocation universelle (Van Parijs 1995; Vanderborght et Van Parijs 2005) a été formulée d'un point de vue républicain, devenant alors «revenu citoyen», par Stuart White (2003). Dans cet article, je m'appuie sur les formulations de White tout en adossant le « revenu citoyen » à d'autres ressources que celles envisagées par White. 
parce qu'il admet plusieurs modalités d'expression de l'égalité ellemême (Parfit 1997).

\section{SOCIALISME LIBÉRAL ET RÉPUBLICANISME : LE TEST DE LA LIBERTÉ}

Si l'humanisme civique définit la liberté politique comme une fin à laquelle tout homme est tenu d'adhérer s'il veut être réellement un homme, dans sa reformulation de la théorie républicaine, Pettit définit la liberté comme un moyen dont la jouissance garantit que des choix individuels peuvent être faits en contexte non-dominé. Mais en quoi cette définition se distingue-t-elle d'une définition plus classique de la liberté que l'on trouve dans le libéralisme? Répondre à cette question est une étape importante de notre démonstration car le libéralisme du «socialisme libéral» semble reprendre plus classiquement les éléments de la définition de la liberté comprise comme non-interférence. C'est donc, dans ce premier moment, du côté de la définition de la liberté que l'on peut distinguer socialisme libéral et républicanisme.

La liberté positive est dite telle dans le sens où tout individu qui en jouit, possède quelque chose de réel (la maîtrise de soi), la liberté négative est négative, classiquement, parce qu'elle se caractérise par une absence: une absence d'interférence. Pour Pettit si la liberté républicaine peut être décrite comme une liberté négative, elle ne doit pas cependant être confondue avec la liberté comme non-interférence. Son objet est l'absence «non pas d'interférence, mais de maitrise exercée par les autres» sur soi (Pettit 2004, p. 41). Le fait que l'on ne soit pas soumis à la maitrise d'un autre (différent du fait d'être maître de soi) est ce que Pettit appelle «la non-domination» (Pettit 2004, p. 41). Cette liberté porte l'accent sur la qualité des relations que les individus peuvent nouer entre eux, et Pettit souligne qu'une absence d'interférence n'est pas automatiquement la marque d'une absence de domination. En ce sens il y aurait bien une liberté négative de type républicain différente de la liberté négative libérale. La première porte l'accent sur le fait de ne pas être dominé, la seconde sur celui de ne pas être gêné dans ses actions ou empêché physiquement d'agir. La liberté républicaine se définit avant tout par son caractère social alors que la liberté libérale 
s'attache aux actions non entravées d'un individu quel que soit le contexte. La liberté républicaine place les institutions au premier plan et non l'individu, si bien qu'elle peut envisager d'accueillir favorablement certaines «entraves » ou interférences, si celles-ci sont jugées non arbitraires.

Mettre l'accent sur l'«arbitraire » permet de préciser ce que recouvre, selon Pettit, le concept de domination en portant la lumière sur une dimension particulière de l'interférence : le caractère seulement possible de cette dernière. Selon Pettit, la définition de l'interférence dans la conception de la liberté comme non-interférence néglige le fait qu'une interférence peut être seulement possible et non réelle, mais aussi le fait qu'une interférence peut ne pas être arbitraire et, dans ce cas, être constitutive de la liberté, même négative. Le travail de Pettit consiste précisément à montrer que des interférences non-abritraires peuvent être nécessaires pour contrer la simple possibilité de l'interférence qui peut miner les relations qu'entretiennent les individus dans une société (autant, selon lui, que peut le faire une interférence réelle). C'est cette configuration que la définition négative de la liberté empêche habituellement d'envisager. En effet, pour cette dernière, un esclave soumis au pouvoir d'un bon maître pourrait difficilement être décrit comme privé de liberté si «être un bon maitre » signifie " ne pas interférer dans les actions de l'esclave ». Le bon maître pourrait même avoir une attitude bienveillante à l'égard de l'esclave, le couvrir de richesses et étendre son champ d'actions possibles, si bien qu'on pourrait voir en lui un vecteur de la liberté de l'esclave comprise comme non-interférence. Pour Pettit le fait qu'il n'y ait pas, dans les relations entre le maitre et l'esclave, d'interférences réelles, n'enlève rien à la situation sociale qui fait qu'un individu a un statut d'esclave et qu'un autre jouit d'un statut de maitre. La relation de domination, même si elle n'est pas actualisée en permanence (et quand bien même le serait-elle jamais) dans un mauvais traitement ou dans des interférences réelles, est toujours susceptible de l'être : il est inscrit dans la relation maitre/esclave que le maitre peut - son statut social l'y autorise - changer de comportement et se révéler un mauvais maître.

En conséquence, il y a des chances pour que cette situation entraîne une attitude de servilité de la part de l'esclave, au moins pour prévenir tout changement d'attitude possible. Bref, l'esclave ne 
jouit pas d'un statut social qui l'autorise à regarder le maître droit dans les yeux.

Selon Philip Pettit, le républicanisme se caractérise ainsi essentiellement par une appréhension de l'individu - et de sa liberté - à partir du statut que lui confère son appartenance à une communauté politique. Dans Républicanisme, Pettit affirme ainsi que le cœur de la philosophie républicaine est constitué par une définition de la liberté comprise comme non-domination, c'est-à-dire comme «position dont jouit un individu quand il vit en présence d'autres personnes qui, en vertu d'un certain dispositif social, s'abstiennent d'exercer sur autrui un pouvoir de domination » (Pettit 2004, p. 95). Cette liberté est donc relationnelle, et suppose l'inscription de l'individu dans un espace politique auquel il participe et qui le protège. Le statut de «citoyen» désigne la forme empirique que prend cette définition abstraite de la liberté. La «non-domination » est donc un idéal politique qui prend la forme d'un idéal communautaire, en ce qu'elle est un bien à la fois social et commun qui nécessite institutions et régulations politiques pour être défendue ${ }^{7}:$ "La liberté comme non-domination exige la liberté dans la cité, pas une liberté dans le désert » (Pettit 2004, p. 162).

Les biens que promeut l'idéal politique libéral - qu'il s'agisse de l'utilité, du bonheur, du soulagement de la misère - sont quant à eux des biens non sociaux et non communautaires, au sens où il est «manifestement possible, en principe, que quelqu'un réussisse bien sur ces plans tandis qu'il en irait tout autrement pour d'autres », tandis qu'« au jeu de la non-domination, nos destins sont intimement liés » (Pettit 2004, p. 161-162). Pour Pettit une telle conception de la liberté, au fondement des conceptions républicaines de l'individu et de la cité, nécessite de « désirer l'égalité républicaine » et de réaliser, en pratique, la « communauté républicaine » (Pettit 2004, p. 166) ${ }^{8}$. La justice et l'égalité sont les conditions de possibilité de la

7. Un bien social, car elle nécessite «l'existence d'un groupe d'individus qui adoptent des attitudes intentionnelles et mènent peut-être des activités intentionnelles ", mais un bien commun par ailleurs, car la poursuite de la non-domination implique qu'elle ne peut être augmentée ou diminuée "pour certains membres d'un groupe sans l'être en même temps pour les autres» (Pettit 2004, p. 160).

8. Sur cette question Pettit renvoie à l'ouvrage de J.-F. Spitz, La liberté politique (Spitz, 1995). 
liberté politique qui rend effective la liberté des individus. Cette articulation des moyens (l'égalité et la justice) et d'une double fin (la liberté politique et la liberté de l'individu) est ce qui se perd dans le socialisme libéral où n'apparait plus le lien étroit entre les outils redistributifs (la justice et l'égalité) et la justification politique de leur utilisation.

Gareth Stedman Jones (2007) a proposé une relecture de l'évolution historique des manières de justifier les projets d'éradication de la pauvreté. Alors que sous la Révolution française, lutter contre la pauvreté revêt une dimension républicaine explicite - il s'agit de doter matériellement les individus pour qu'ils puissent exercer effectivement leur citoyenneté - cet aspect est marginalisé dans des projets en apparence identiques de la fin du XIX ${ }^{\mathrm{e}}$ siècle. L'État social devient un enjeu technique de soulagement de la misère (et aussi, comme ce fut exemplairement le cas en Allemagne, de maitrise des débordements populaires), non un projet politique inclusif?.

Ces conceptions différentes de la liberté se doublent de manières distinctes d'appréhender les formes de justification de l'intervention publique ou sociale dans le jeu économique. Un certain nombre de propositions - solidarisme, formes d'impôts, rôle d'assistance de l'État, promotion de la propriété sociale, mutuellisme, etc. présentes dans la tradition socialiste libérale (Audier 2006) peuvent évidemment être accueillies par la tradition républicaine, mais, aux yeux de cette dernière, à la condition seulement qu'elles puissent justifier de la promotion de l'idéal politique républicain de liberté comme non-domination. Plus encore le rôle du marché peut être valorisé aussi bien dans un cadre socialiste-libéral que républicain, mais là encore les justifications convoquées vont diverger : dans le

9. Par exemple, si Thomas Paine, dans Justice agraire (Paine, 1995) et Henry George, dans Progrès et pauvreté (George, 1925), semblent proposer des solutions proches techniquement, elles n'en relèvent pas moins d'une forme de justification différente, politique dans le premier cas, purement sociale dans le second. L'égalitarisme matériel qu'ils défendent en partie l'un et l'autre est conçu dans le cas de Paine comme une manière d'accèder à une autonomie matérielle, condition de possibilité de l'exercice de la citoyenneté et de l'inscription de tout individu dans la cité. Chez George, il s'agit d'une application stricte de la justice «naturelle» (la terre est, de droit naturel, la propriété de tous), dont la visée reste attachée à l'individu en lui-même et pour lui-même, dont il s'agit de concevoir la sortie de l'état de pauvreté (Stedman Jones 2007, p. 150-167). 
premier cas, le fait que le marché soit à l'avantage des plus défavorisés sera mis en avant, dans le second, il faudra s'assurer de la compatibilité du marché et de la promotion de la liberté comme non-domination. C'est cet aspect que nous abordons dans la section suivante.

\section{LE MARCHÉ DES ÉGAUX? PROMOTION DE LA NON DOMINATION ET FONCTIONNEMENT DU MARCHÉ RÉPUBLICAIN}

Dans Républicanisme, Pettit soutient à la fois que l'idéal républicain est compatible avec un objectif de "prospérité économique » (Pettit 2004, p. 214-217) et avec de nombreux attendus socialistes (Pettit 2004, p. 184-188). Cette double compatibilité tient à l'objectif visé par un gouvernement républicain : la promotion de l'idéal de nondomination. En effet, d'un point de vue républicain, la prospérité économique ne justifie pas tout. Le seul horizon de l'efficiency ne saurait tenir lieu de cap au gouvernement républicain. Ce n'est que dans la mesure où cette efficacité permet de mieux garantir un contexte favorable à la non-domination (aussi bien du point de vue extensif qu'intensif) qu'elle peut être favorablement accueillie par le républicanisme. Pettit considère néanmoins qu'une "politique économique républicaine» (Pettit 2004, p. 215) ne saurait être décrite en dehors des débats économiques concrets qui surgissent dans une société donnée et au cours desquels le point de vue républicain trouvera à s'exprimer. Il n'y aurait pas, selon lui, d'architecture proprement républicaine de l'économie, et, en ce sens, l'idée d'un marché républicain n'est pas intégrée au projet social et politique du républicanisme tel qu'il le défend. Ce sont plutôt des correctifs républicains au marché que le républicanisme serait appelé à mettre en avant. Malgré tout, nous l'avons noté à l'instant, Pettit affirme la compatibilité du projet républicain et du projet socialiste, or ce dernier se définit essentiellement comme une redéfinition du fonctionnement économique de la société. Pour Pettit, il est probable que l'idéal de la liberté comme nondomination est en réalité le but que poursuit le renversement du mode de fonctionnement capitaliste de la société, en aucun cas ce renversement n'est un idéal en soi. C'est bien, affirme Pettit, pour 
accéder à des formes d'autonomie dans le travail et ne plus être placés dans "un état de sujetion vis-à-vis de leurs maîtres » que nombre d'ouvriers embrassèrent l'idéal socialiste dès la fin du $\mathrm{XIX}^{\mathrm{e}}$ siècle. En ce sens, sans en retenir forcément les moyens, le républicanisme peut partager une même fin que celle qui pousse certains à adopter l'idéal socialiste. J'aimerais suggérer au contraire que certains moyens "socialistes » sont en fait rendus nécessaires par les exigences mêmes que l'idéal républicain fait peser sur le marché et son fonctionnement.

Dans la mesure où le marché suppose des agents libres, propriétaires, susceptibles d'échanger librement leurs biens ou services, qu'est-ce qui pourrait autoriser, en effet, des formes d'intervention dans ce système que Smith décrivait comme un « système de la liberté naturelle »? Pour Pettit aucun de ces éléments constitutifs du fonctionnement d'un marché standard ne sont en eux-mêmes contradictoires avec l'idéal de liberté comme nondomination. La propriété privée, par exemple (et la tradition républicaine témoigne historiquement d'un attachement à cette dernière), est tout à fait acceptée par les républicains jusqu'au point où les inégalités de propriété (le fait que certains soient propriétaires et d'autres non ou que certains le soient plus que d'autres) pourraient se révéler être des instruments de domination des propriétaires envers les non-propriétaires. Si ce devait être le cas, suggère Pettit, alors « cela suffirait à justifer des ajustements institutionnels », c'està-dire des interférences non-arbitraires dans le jeu du marché. Toute la question revient donc à déterminer ce «point» de retournement. Le reproche d'indétermination qui a pu être fait au concept de «domination»(McMahon 2005), pourrait se voir ici reconduit: pourquoi, en effet, ne pourrait-on pas se sentir «dominé » par tout individu possédant plus de biens que soi ? Pourquoi le fait qu'autrui possède des titres de propriété que l'on ne possède pas soi-même ne justifierait-il pas que l'on puisse se sentir dominé et, par conséquent, qu'une intervention publique vienne légitimement corriger cette situation?

Pettit propose, pour sortir de ce dilemme, de distinguer les situations dans lesquelles la liberté comme non-domination est « compromise » de celles dans lesquelles elle est seulement « conditionnée ». Il est possible, suggère-t-il, que les choix qui s'offrent aux individus dans une société aux propriétés inégalement réparties, 
soient d'une nature qui tienne à cette distribution inégale (les choix de tous les individus sont donc en partie conditionnés par la structure de la distribution des propriétés ou des richesses au sein de cette société). Mais qu'ils soient conditionnés, ne veut pas dire pour autant que, pour les choix qui sont accessibles à un individu, même démuni, une parfaite autonomie dans la décision ne soit pas garantie (notamment par un réseau d'institutions républicaines qui veillent à ce que ce soit le cas). En ce sens, la liberté comme non-domination est certes conditionnée, mais non compromise par cette distribution inégalitaire. Si cette distinction permet de nuancer l'extension que peut revêtir le critère de domination, elle ne répond pas totalement à la question de savoir comment on peut reconnaitre le passage de l'inégalité (matérielle) des possessions à la domination. Pourtant, c'est au nom de ce passage seulement qu'une intervention est justifiable (car elle sera jugée alors non-arbitraire). Pettit soutient de son côté qu'un «égalitarisme structurel » est suffisant pour prévenir la transformation de l'inégalité en domination (2004, p. 156-158; 2006, p. 141). Il n'y a donc pas, dans le cadre de la théorie républicaine défendue par Pettit, d'horizon égalitariste matériel qui puisse être considéré comme un but désirable en soi, ni même comme un moyen nécessaire à la promotion de la non-domination. Malgré cette insistance, Pettit n'en reconnaît pas moins que des inégalités trop étendues donnent bien lieu à domination. L'analyse que propose Pettit de la relation marchande, pour déterminer si on peut voir en elle un obstacle à la liberté comme non-domination, permet d'éclairer cette contradiction apparente.

Le marché est présenté classiquement comme le lieu de rencontre d'agents qui échangent des biens ou des services, non pas sous la contrainte ou la menace des uns envers les autres, mais en vue de satisfaire chacun leurs intérêts. Suivant l'exemple que prend Pettit (2006, p. 143) une situation d'échange marchand peut se comprendre exemplairement comme une situation où un individu qui a devant lui trois choix possibles $\{A, B, C\}$ se voit proposer contre une récompense de choisir l'une de ces options, $A$ par exemple. L'offre d'échange modifie donc son ensemble initial le faisant passer de $\{A, B, C\}$ à $\{A, A$, $B, C\}$, où $A^{\prime}$ correspond à $A$ plus la récompense. On pourrait envisager que la récompense agit comme une "contrainte» rendant le choix $A$ ' en quelque sorte « nécessaire ». Cette contrainte, souligne toutefois Pettit, ne saurait 
être appréhendée comme une diminution de la liberté comme nondomination, il s'agit d'un conditionnement de la liberté certes, mais en aucun cas d'une diminution de cette dernière: je peux bien choisir encore $C$ ou $B$, voire même $A$ (refusant la récompense qui m'est offerte en A' pour marquer que mon choix pour A se fait en toute indépendance). Pour Pettit, on ne saurait donc confondre cette situation avec une autre où, devant les mêmes choix possibles $\{A, B, C\}$, le même individu se verrait menacer, s'il choisit $C$, de subir une pénalité. Dans ce cas, la pénalité empêche d'envisager que $C$ puisse être voulu sans la sanction qui l'accompagne. Dans cette dernière situation, l'ensemble des choix est modifié en $\left\{A, B, C^{\prime}\right\}$, et le fait qu'un individu puisse modifier l'ensemble de mes choix de façon arbitraire est un signe manifeste de domination. La liberté n'est alors pas seulement conditionnée mais diminuée dans un tel cas de figure. Une relation marchande idéalement (et c'est ainsi qu'elle est présentée par les défenseurs libéraux du marché) correspond à la première situation, l'offre marchande ne saurait donc être confondue avec une menace, il faudrait plutôt y voir une forme d'incitation préservant l'accessibilité des choix et la capacité de contrôle (Pettit 2006, p. 144) ${ }^{10}$. En ce sens, «l'offre d'une récompense de marché est toujours une offre que l'on est susceptible de refuser» (Pettit 2006, p. 143), elle laisse à celui qui la reçoit le contrôle sur la situation ${ }^{11}$.

Partant d'une description idéale du marché (y compris aux yeux de ses défenseurs libéraux), Pettit souligne que la liberté comme non-domination peut trouver à s'y épanouir, notamment parce qu'un marché ainsi conçu, fonctionnant à la prime plus qu'à la sanction, laisse à chaque acteur un contrôle complet sur la situation d'échange. La théorie républicaine propose donc un idéal politique

10. Il est à noter que c'est ici la structure du choix initial qui est annulée. Dans le cas précédent, cette structure était préservée, même si augmentée d'une option possible. C'est sur ce point que Jean-Fabien Spitz (2010, p. 173-178) se montre le plus critique : la nature de l'option ajoutée dans la structure des choix peut être telle, selon lui, qu'elle ne se distingue pas réellement du contre exemple, celui de la sanction, décrit par Pettit.

11. Il y a là un tour de force démonstratif de Pettit, qui semble inviter à penser qu'idéalement le marché et les relations qui s'y jouent ont des affinités fortes avec la définition de la liberté comprise comme non-domination. Par là, il s'oppose à ceux qui voient dans l'application du républicanisme à l'économie de marché une tentative dangereuse (Gaus 2003). 
qui permet de justifier le marché, en régulant ce dernier pour qu'il soit le lieu d'épanouissement d'une liberté comprise comme nondomination plutôt qu'une liberté comprise comme non-interférence, d'une liberté répulicaine plutôt que libérale. La régulation consisterait alors à cibler toutes les formes de compromission de la liberté, dans des échanges tels que la structure des choix serait aux mains de l'un des contractants et d'un seul. Ainsi redéfini, le marché fonctionnera sous contrainte républicaine, comprenant des « restrictions sur certaines activités », et une "redistribution des propriétés », l'impôt étant l'instrument de l'une et l'autre de ces mesures (Pettit 2006, p. 147). La liberté comme non-domination, choisie comme critère d'évaluation de l'échange, permet d'introduire des interventions publiques dans ce dernier sans le dénaturer: «En perpétrant des interférences publiques, l'État peut être une présence non-arbitraire qui conditionne mais ne compromet pas la liberté des gens ». Par là même l'Etat prévient les tendances à la domination de certains et non seulement la réalisation de telles tendances (Pettit 2006, p. 147). Une telle stratégie renforce, aux yeux de Pettit, plutôt qu'elle ne s'y oppose, le fonctionnement du marché (idéal) et la promotion de la liberté comme non-domination.

La description d'un marché ainsi conçu, de même que les conclusions pratiques qu'il lui applique (sous la forme de régulations étatiques), dénotent la prédilection que Pettit a constamment manifestée, en matière de promotion de la non-domination, pour la voie constitutionnelle plutôt que pour celle de la réciprocité des pouvoirs. La « réciprocité des pouvoirs » n’implique pas seulement qu'un État corrige ou régule le fonctionnement du marché, mais que chaque citoyen soit doté d'attributs individuels qui lui permettent de se tenir dans un rapport d'égal avec ses concitoyens, elle vise à « égaliser les ressources dont disposent le dominant et le dominé, de sorte, idéalement, qu'une personne antérieurement dominée puisse parvenir à se défendre elle-même de toutes interférences engendrées par le dominant» (Pettit 2004, p. 95). Cette stratégie parait à Pettit peu réaliste et coûteuse socialement. Il privilégie plutôt une architecture institutionnelle permettant d'empêcher qu'un individu en situation de domination puisse utiliser son pouvoir à l'encontre d'un autre. Il s'agit de contenir le pouvoir de nuire de certains : une autorité constitutionnelle, note-t-il, "éradique la domination que certains individus peuvent exercer sur d'autres et, dans la mesure où 
elle n'exerce pas elle-même de domination sur les autres, [elle] mettra fin à toute domination » (Pettit 2004, p. 96). Pourtant, dans le cas de relations marchandes, il semble que la stratégie de réciprocité des pouvoirs pourrait être mieux adaptée pour lutter contre les formes de domination qui peuvent s'y déployer, mais surtout, dans la logique même que promeut Pettit de rapporter le républicanisme et ses principes aux idéaux du marché, mieux adaptée pour permettre un fonctionnement souple de ce même marché, sans qu'il ne soit entravé par des règles et des interventions publiques permanentes. En ce sens, étant donné l'écart entre le marché idéal et la réalité de son fonctionnement, cette stratégie pourrait bien être, dans ce cas précis, plus réaliste et moins coûteuse socialement.

Dans l'analyse du marché qu'il propose, Pettit nous invite en effet à accepter comme présupposé que les «déséquilibres en matière de propriété et de pouvoir qui donnent aux échanges de biens et de services leurs formes n'ont pas l'effet d'autoriser la domination au sein des échanges marchands » (Pettit 2006, p. 142). Une manière de s'assurer véritablement qu'ils n'aient pas cet effet peut consister à doter les individus de ressources matérielles suffisantes, afin de leur permettre de se soustraire à l'échange si besoin (de le refuser, comme dirait Pettit). Ainsi pour que le face-à-face entre individus, guidés par leurs intérêts, puisse toujours se produire de façon fluide, et pour autant "entre égaux», il est sans doute préférable de privilégier une stratégie de réciprocité des pouvoirs, dont le mécanisme d'allocation universelle et inconditionnelle d'un « revenu citoyen » pourrait être une bonne application (White 2003). Il s'agit donc, par ce biais, de mettre en avant un égalitarisme matériel auquel Pettit refuse d'accorder une place dans son républicanisme. J'aimerais suggérer qu'au moins une manière de justifier cet égalitarisme matériel peut en définitive le ramener dans le giron d'une définition de l'égalitarisme structurel. Il s'agit de défendre une forme d'égalitarisme matériel qui ne soit pas synonyme d'une égalité stricte du point de vue des possessions entre tous les individus, mais d'une égalité quant à la possession d'un certain nombre de 
ressources matérielles permettant d'assurer l'indépendance matérielle - et par voie de diffussion, politique - des individus ${ }^{12}$.

\section{QUEL ÉGALITARISME MATÉRIEL? UN ASPECT SOCIALISTE DU MACHÉ RÉPUBLICAIN}

Faire du revenu de citoyenneté un socle égalitaire autorisant, comme l'écrit White, « une réciprocité honnête » ou, comme je l'ai suggéré, "un marché des égaux» peut donner lieu à une critique classique de la part des partisans libéraux du marché : l'idée de doter de ressources identiques (ou du moins d'un socle identique de ressources) les agents est généreuse, mais comment la financera-ton? Comment peut-on promouvoir l'égalité en puisant dans les richesses des uns pour les donner à d'autres sans sacrifier la liberté? Pour répondre à ces doutes, il faut imaginer sur quelles ressources communes les acteurs d'un marché libre vont pouvoir s'appuyer pour échanger en égaux ${ }^{13}$. La réponse la plus traditionnelle, du côté du socialisme, fut de considérer l'appropriation par l'État des moyens de production, d'envisager en somme une propriété publique des entreprises, sans que cette dernière ne soit incompatible avec un mécanisme de "marché » pour la distribution des produits comme l'ont montré des développements contemporains du socialisme de marché (Roemer 1996, p. 287-302; Shleifer et Vishny

12. En ce sens l'égalité matérielle défendue dans cet article est une égalité matérielle du "socle de base », celui qui permet l'expression de soi et la manifestation de ses désirs, qui rattache aussi les individus les uns aux autres au sein d'une même communauté politique. Un socle, enfin, sur lequel des disparités légitimes vont pouvoir émerger, réintroduisant des formes de richesse inégales au sein de la communauté mais dans la stricte mesure où ces dernières ne remettent pas en cause l'indépendance des membres de cette communauté. Cette indépendance n'est pas justifiée seulement dans les termes de la possibilité pour chacun de poursuivre ses propres désirs, mais, aussi, dans ceux, politiques, d'une garantie à la bonne participation de tous à la vie de la cité. Je remercie un commentateur anonyme d'avoir attiré mon attention, à ce stade du raisonnement, sur ces distinctions.

13. Ce travail est évidemment éminemment politique, il suppose de repenser nos manières de combiner les choses et les êtres, de travailler sur la matière de nos sociétés et des hommes qui les composent pour en extraire des formes de vie commune plus épanouissantes pour chacun. Une première tentative est proposée, à la lumière de la philosophie « artisanale » de Platon, par Arnaud Macé (2010). 
1994, p. 165) ${ }^{14}$. Telle n'est pas la vision du socialisme libéral qui propose de son côté de socialiser pour partie le produit du travail humain, sous la forme de transferts sociaux, sans toucher à la propriété privée des moyens de production.

D’une manière générale, les approches républicaines de l'économie considèrent que la propriété privée n'a pas à être condamnée mais, au contraire, qu'elle doit être l'objet d'une meilleure répartition dans la société dans la mesure où le titre de propriétaire renforce le contrôle que les individus peuvent avoir sur leur existence et donc leur capacité à se gouverner eux-mêmes, en particulier en tant que travailleurs (Sunstein 1997; et surtout Simon 1991). En conséquence de cette valorisation de la propriété, les républicains ont toujours privilégié la pleine propriété individuelle des fruits du travail personnel, du moins à condition que chacun soit mis dans une situation de départ jugée égale, seule garante du mérite que chacun peut revendiquer des justes bénéfices de ses actions (Spitz 2005). Dans un tel schéma, les transferts sociaux peuvent vite être perçus comme des formes d'intrusion de la puissance publique dans la sphère individuelle. Comment parviendra-ton, dès lors, à réaliser l'égalité des conditions sans sacrifier la liberté des individus?

C'est sans doute chez un économiste et républicain du $\mathrm{XIX}^{\mathrm{e}}$ siècle, Léon Walras, qu'on trouve la formulation la plus précise de l'idée que des ressources communes ne sont pas forcément des ressources prises à certains en vue de les transformer en un fonds commun, mais des ressources qui appartiennent, par nature, à tous et ne sauraient être appropriées de façon exclusive par quiconque (Walras 1990; Bourdeau 2006). Une communauté naturelle ou objective fonde dès lors la communauté politique et permet l'égalisation matérielle des conditions. Walras souligne qu' [i]l y a ainsi deux espèces de richesse sociale à répartir : les terres et les facultés personnelles», distribuées entre «deux types sociaux», "l'État et l'individu»: «[]e principe de l'inégalité des positions exige que les

14. Shleifer et Vishny notent ainsi qu'un «marché socialiste » est une « économie dans laquelle les firmes sont possédées et contrôlées par le gouvernement mais dont les produits sont vendus aux consommateurs sur des marchés compétitifs » (Shleifer \& Vishny 1994, p. 165). 
facultés personnelles soient attribuées à l'individu, et le principe de l'égalité des conditions exige que les terres soient attribuées à l'État. » (Walras 1992, p. 421-422). Il s'agit là d'une manière d'attirer l'attention, comme le dit Williams, sur une égalité qui existe d'ores et déjà mais qui est «obscurcie ou négligée par les arrangements sociaux actuels » (Williams 2006, p. 452). Si Williams suggère qu'un égalitarisme strict repose sur la valeur d'égal respect qu'il faudrait accorder à tous - privilégiant en ce sens le «point de vue humain»- le contrôle et le bénéfice à parts égales des ressources naturelles introduirait quelque chose comme un «point de vue naturel» qui aurait cette fois tout à voir avec l'égalité politique. Mais de la même manière que l'égalité de respect accorde moins d'importance aux structures qui distribuent de façon légitime le prestige ou les statuts sociaux, contrairement à ce qu'une théorie de l'égalité des chances ou des opportunités invite à faire, cette égalité matérielle peut être déconnectée de la question des inégalités qui surviennent dans le jeu des échanges économiques et sociaux une fois ce socle égalitaire assuré.

Par contraste, le socialisme libéral ne présente en réalité aucune doctrine cohérente de l'égalité. Ce qu'il revendique sous le nom d'égalité sociale, c'est en fait une forme de protection accordée aux plus démunis. Il est en réalité prioritariste, et non égalitariste : la position prioritariste, rappelle Parfit, suggère que des avantages doivent être accordés aux individus dans la mesure où ils occupent une position jugée par elle-même défavorable (Parfit 1997). Ainsi, le prioritarisme doit être distingué de l'égalitarisme. Selon Parfit, la confusion entre égalitarisme et prioritarisme concerne les biens ou les distributions de biens qui ne peuvent faire l'objet d'une distribution égale sans heurter un sens de la justice distributive (au sens d'Aristote) qui fait appel à une prise en compte des circonstances, ou de ce qui, pour chaque individu, relève de sa complexion propre (les soins de santé aux personnes malades par exemple). Ainsi Parfit note-t-il que si la distinction a longtemps passé inaperçue, cela tient au fait que les combats égalitaristes dans les siècles passés ont porté sur des demandes pour lesquelles elle était non pertinente : ce qui était revendiqué alors c'était " une égalité civile ou politique », s'attaquant "aux privilèges arbitraires, ou aux différences de statuts» (Parfit 1997). L'égalitarisme matériel associé à une distribution égale des ressources naturelles est une manifestation particulière de 
l'égalité structurelle ou de statut revendiquée par Pettit, en ce sens cet égalitarisme relève de ces objectifs égalitaires que Parfit juge déjà réalisés ${ }^{15}$.

Les revenus de l'État, issus d'une richesse commune objectivement déterminée (celle de la terre qui, non produite, appartient également à tous et qui, parce que rare, est bien une richesse et non un "don» gratuit de la nature), sont transformés dans l'optique walrassienne en services publics qui permettent de réaliser la solidarité au fondement de la communauté. On pourrait envisager que cette traduction en services publics soit remplacée par ou couplée à l'octroi d'un capital (Paine 1995; Ackerman et Alstot 1999) ou d'un revenu (White 2003) pour tous les citoyens afin de les «armer» en vue de faciliter leur participation au jeu du marché, ou, au contraire, leur retrait de ce même jeu si besoin (Arnsperger 2009, p. 158-162) ${ }^{16}$.

Quoiqu'il en soit, le marché, tel qu'envisagé par Léon Walras, comporte bien un volet égalitariste matériel justifié par un objectif républicain : la propriété publique des ressources naturelles en vue de favoriser une citoyenneté effective. Ainsi, la condition de l'égalitarisme matériel (la propriété publique) se confond avec la définition même du citoyen, puisque c'est au titre de citoyen (membre de l'État) que tout individu a droit à une part du revenu de l'État, revenu qui est lui-même la manifestation du caractère commun des richesses naturelles ${ }^{17}$.

L'objectif d'égalité est souvent associé à la montée d'une instance centralisée, corrigeant les écarts, et donc, en dernier lieu, les causes

15. Il y a donc bien une dimension téléologique (au sens de Parfit) de l'égalitarisme matériel des ressources, rencontrant le principe d'égalité qui veut qu'il soit « en soi mauvais que certains individus soient moins bien lotis que d'autres» (Parfit 1997). Ce serait le cas au point de vue des ressources naturelles.

16. D'autres solutions sont envisageables qui répondraient à une logique identique : la suppression de l'héritage, ainsi que la revendiquaient pour les mêmes raisons de «mérite» ou «non-mérite » personnel les saints-simoniens du XIX e siècle, la taxation des intérêts ou des rentes, etc.

17. Si la prespective walrassienne s'appuie exclusivement sur un langage du droit naturel de l'État et un idéal républicain, le contexte actuel de la crise écologique incite à considérer avec bienveillance l'option qu'il met en avant, y compris lorsqu'on ne partage pas cet idéal républicain. La promotion d'une propriété et d'un contrôle publics des ressources naturelles pourrait être défendue du point de vue de l'intérêt écologique qu'il y aurait à voir les ressources naturelles être publiquement possédées et contrôlées. 
de ces écarts, c'est-à-dire les actions mêmes des individus appartenant à la communauté politique (Walzer 1997, p. 14). Le socialisme libéral, par les mesures redistributives qu'il met en avant se situe clairement du côté d'une distribution expost de la richesse du côté d'une redistribution. Si le revenu d'existence financé par les ressources communes a pu connaitre une certaine faveur chez les républicains radicaux du XVIII ${ }^{\mathrm{e}}$ siècle, de Condorcet à Paine, l'une des raisons avancée était qu'un tel mécanisme permettait un équilibrage ex ante de la relation marchande, économisant de trop nombreuses irruptions de l'Etat dans les relations marchandes et dans la circulation de la richesse, par le jeu d'une répartition primaire de la richesse. Par là, jugeaient-ils, l'individu se voyait préservé de toute interférence redistributive de l'Etat, jugée abusive, voire arbitraire (Rothschild 2001).

J'ai cherché dans cet article à justifier l'idée d'une architecture proprement républicaine du marché : par définition le marché organise la rencontre d'individus qui ont des biens ou services à s'échanger; dans cette relation, il est probable que les atouts personnels de chacun jouent un rôle non négligeable dans la détermination de l'échange, ce qui plaide pour une stratégie dite de la réciprocité des pouvoirs plutôt que pour la seule stratégie des « dispositifs constitutionnels ». Au nom de cette stratégie, le républicanisme peut et doit promouvoir certaines formes d'égalitarisme matériel. L'architecture du marché républicain que je propose ressemble davantage, j'en ai conscience, à une pièce d'un puzzle plus vaste qu'on pourrait appeler « économie politique républicaine ». Le marché républicain ne serait qu'une dimension de cette dernière, qui comprendrait sans doute une organisation du travail sous la forme d'associations coopératives (Simon 1991; Fleurbaey 2006; Sandel 1996) et nécessiterait peut-être, du côté du marché ainsi refaçonné, que soient facilitées des formes d'échange d'estime, dans la mesure où coopération et estime participent à la promotion de la nondomination (Brennan et Pettit 2004). Ces derniers aspects s'écartent de l'objectif que je m'étais donné dans cet article, à savoir étayer - d'un point de vue normatif - l'idée d'une architecture proprement 
républicaine de l'échange, architecture qui aurait comme mérite d'ouvrir l'espace des possibles pour ces autres pans de l'économie politique républicaine dans la mesure où le socle égalitaire garantit à tous un statut économique identique garant d'une liberté vis-à-vis du marché lui-même ${ }^{18}$. J'ai voulu montrer par là que tout accommodement au marché, dans le cadre d'un discours politique, suppose des justifications en termes de buts visés, et qu'il n'est pas certains que ces buts soient clairement identifiés par ceux qui se revendiquent aujourd'hui du «socialisme libéral». Il m'a paru nécessaire de complexifier la manière dont le républicanisme appréhende - du moins chez Pettit - le marché en suggérant que les contraintes que font peser sur lui les régulations républicaines institutionnelles en modifient fortement la nature, au point qu'une autre stratégie peut s'avérer plus pertinente aussi bien en termes de promotion de la non-domination, qu'en termes d'efficacité du marché. Cette stratégie de réciprocité des pouvoirs engage une architecture spécifiquement républicaine du marché en ce qu'elle rend nécessaire, ex ante, une forme d'égalité matérielle des acteurs dont la mise en œuvre relève à proprement parler d'un moment socialiste du républicanisme. Ce travail de clarification des raisons (républicaines) qu'il convient d'avancer pour justifier l'existence du marché s'est limité à l'évaluation de la cohérence des moyens à mettre en œuvre pour atteindre l'objectif d'une promotion de la non-domination. Il resterait à confronter un tel programme au test de sa réalisation pratique d'un côté, et à le situer dans le champ des théories de l'égalité contemporaines de l'autre ${ }^{19}$.

18. Ainsi qu'il jouisse ou non d'estime, qu'il appartienne ou non à une coopérative (dans laquelle il pourrait certes mieux manier les instruments d'un gouvernement de soi), tout individu possède un statut d'égal, par la simple appartenance à la communauté politique, qui se traduit par un droit à d'égales ressources matérielles. L'architecture du marché ainsi conçue atténue le phénomène d'une "propriété social-républicaine [qui] accomplit une égalité intrapolitique seulement au prix d'une inégalité extrapolitique» (Simon 1991, p. 1386).

19. Il n'est en effet pas certain que cet égalitarisme matériel soit le seul aspect « égalitaire» que devrait viser une théorie républicaine de l'égalité, j'ai d'ailleurs tenté de suggérer que cet égalitarisme matériel s'insérait dans l'égalitarisme structurel défendu par Pettit plutôt qu'il ne s'y substituait. Pour une bonne traversée des débats les plus récents sur l'égalité, où est cependant absente la théorie républicaine, voir : Knight (2009). 


\section{RÉFÉRENCES}

ACKerman Bruce et Anne Alstot. 1999. The Stakeholder Society. New Haven \& London: Yale University Press.

ARnSPERger Christian. 2009. Éthique de l'existence post-capitaliste. Pour un militantisme existentiel. Paris : Éditions du Cerf.

AUdiER Serge. 2006. Le socialisme libéral. Paris : La Découverte.

BOURDEAU Vincent. 2006. "Les républicains du XIX e siècle étaient-ils des libertariens de gauche? L'exemple d'Auguste et Léon Walras ». Raisons Politiques, vol. 3 n²3, p. 93 108.

Brennan Geoffrey et Philip PetTit. 2004. The Economy of Esteem. An Essay on Civil and Political Society. New York : Oxford University Press.

CANTO-SPERBER Monique et Nadia URBINATI. 2003. Le socialisme libéral. Une anthologie: Europe - Etats-Unis. Paris : Editions Esprit.

DuRKHEIM Émile. 1992. Le Socialisme. Sa définition - Ses débuts - La Doctrrine SaintSimonienne. Paris : Presses Universitaires de France, (1 re édition 1928).

DwORKIN Ronald. 2000. Sovereign Virtue: The Theory and Practice of Equality. Cambridge, MA : Harvard University Press.

FLeUrbaeY Marc. 2006. Capitalisme ou démocratie ? L'alternative du XXI e siècle. Paris : Grasset.

GAuS Gerald F. 2003. "Backwards Into the Future: Neo-Republicanism as a PostSocialist Critique of Market Society ». Social Philosophy and Policy, n²0, p. 59-91.

GeOrge Henry. 1925. Progrès et pauvreté. Enquête sur la cause des crises industrielles et de l'accroissement de la misère au milieu de l'accroissement de la richesse. Le remède. Paris; Bruxelles : Librairie Felix Alcan \& Librairie Vve F. Larcier, (1 re édition 1879).

KNIGHT Carl. 2009. Luck egalitarianism. Equality, Responsability, and Justice. Edinburgh: Edinburgh University Press.

MACÉ Arnaud. 2010. L'atelier de l'invisible. Apprendre à philosopher avec Platon. Alfortville: éditions è̊e / Collection « Chercheurs d'ère».

MCMAHON Christopher. 2005. "The Indeterminacy of Republican Policy». Philosophy \& Public Affairs, n³3, p. 67-93.

PAINE Thomas, éd. Mark PHILP. 1995. Agrarian Justice. In Thomas Paine, The rights of man, Common sense, and other political writings. Oxford: Oxford University Press, (1 re édition 1797).

PARFIT Derek. 1997. «Equality and Priority ». Ratio, n¹0, p. 202-221.

PETTiT Philip. 2004. Républicanisme. Une théorie de la liberté et du gouvernement. Paris: Gallimard, (trad. par Patrick Savidan et Jean-Fabien Spitz de Republicanism, 1997).

PETTIT Philip. 2006. «Freedom in the Market». Politics, Philosophy \& Economics, vol. $5 \mathrm{n}^{\circ} 2$, p. 131-149.

PeTTIT Philip. 2007. «Préface». In Vincent Bourdeau et Roberto Merrill (dir.), La République et ses démons. Essais de républicanisme appliqué. Alfortville: Éditions èße / Collection « chercheurs d'ère ».

POCOCK John G.A. 1997. Le Moment machiavélien: La pensée politique florentine et la tradition républicaine atlantique. Paris: Presses Universitaires de France, (trad. par Luc Borot de The Machiavellian Moment, 1975).

RAWLS John. 1989. Théorie de la justice. Paris : Seuil, (trad. par Catherine Audard de Theory of Justice, 1971,). 
ROEMER John E. 1996. Egalitarian perspective. Essays in Philosophical Economics. Cambridge : Cambridge University Press.

RothsCHILd Emma. 2001. Economic Sentiments. Adam Smith, Condorcet and the Enlightenment. Cambridge (MA); Londres (G.-B.) : Harvard University Press.

SANDEL Michael. 1996. Democracy's Discontent. Amercia in Search of a Public Philosophy. Harvard : The Belknap Press of Havard University Press.

SHLEIFER Andrei et Robert W. WISHNy. 1994. "The Politics of Market Socialism». Journal of Economic Perspectives, vol. 8 n² 2 , p. 165-176.

SIMON William H., 1991. "Social-Republican Property ». UCLA Law Review, vol. 38 nº, p. $1335-1414$.

SPITZ Jean-Fabien. 1995. La Liberté politique. Essai de généalogie conceptuelle. Paris : Presses Universitaires de France.

SPITZ Jean-Fabien. 2005. Le Moment républicain en France. Paris : Gallimard.

SPITZ Jean-Fabien. 2010. «Le marché est-il une institution républicaine?». Diacritica. Revista do centro de estudo humanísticos. Série filosofia e cultura, vol. 24 n² 2 , p. 165-192.

STEDMAN JONES Gareth. 2007. La Fin de la pauvreté? Un débat historique. Alfortville: éditions è̊e / Collection «Chercheurs d'ère », (trad. par Vincent Bourdeau, François Jarrige et Julien Vincent de An End to Poverty? A Historical Debate).

SunsteIn Cass R. 1997. Free Markets and Social Justice. New-York; Oxford: Oxford University Press.

VANDERborght Yannick et Philip VAN PARIjs. 2005. L'allocation universelle. Paris : La Découverte.

VAN PARIJS Philip. 1995. Real Freedom for All: What (if anything) can justify capitalism? Oxford : Oxford University Press.

WALRAS Léon. 1990. Études d'économie sociale: théorie de la répartition de la richesse sociale. Euvres Économiques Complètes, Vol. IX. Paris : Economica, (1 re édition 1896).

WALRAS Léon. 1992. «Esquisse d'une doctrine économique et sociale », (1 re édition 1898). In CEuvres Économiques Complètes, vol. X Études d'économie politique appliquée : théorie de la production de la richesse sociale. Paris : Economica.

WALZER Michael. 1997. Sphères de justice. Une défense du pluralisme et de l'égalité. Paris : Seuil, (trad. par Pascal Engel de Spheres of Justice, 1983).

White Stuart. 2003. The Civic Minimum: On the rights and obligations of economic citizenship. Oxford : Oxford University Press.

WiLliams Bernard. 2006. "The Idea of Equality», (1 re édition 1979). In Robert E. Goodin et Philip Pettit (ed.), Contemporary Political Philosophy. An Anthology. Malden (E.-U.) ; Oxford (G.-B.) ; Victoria (Aus.) : Blackwell. 